\title{
MEDIACIÓN DIGITAL Y MEMORIA HISTÓRICA: UNA MIRADA DESDE NAVARRA
}

\section{DIGITAL MEDIATION AND HISTORICAL MEMORY: AN APPROACH FROM NAVARRA}

\author{
Álvaro Baraibar* \\ Universidad Pública de Navarra. España
}

\begin{abstract}
RESUMEN: El presente artículo pretende acercarse al papel que la mediación digital y las Humanidades Digitales tienen en la gestión del patrimonio de la memoria histórica en sus diferentes expresiones. La mirada parte de Navarra, territorio objeto de una importante represión en los meses y años inmediatos al golpe militar de 1936, y dialoga con otras experiencias internacionales. El trabajo pretende, además, una reflexión sobre la oportunidad de estos nuevos desarrollos tecnológicos teniendo presente el objetivo de reconocimiento y reparación de las víctimas, así como la visibilización y preservación de su memoria en una sociedad post-conflicto como la navarra, pero extrapolable a otros contextos geográficos y cronológicos.
\end{abstract}

PALABRAS CLAVE: Memoria Histórica, Lugares de Memoria, mediación digital, Humanidades Digitales.

\begin{abstract}
The present article makes an attempt to approach the role that digital mediation and Digital Humanities have in the management of the heritage of historical memory in its different expressions. The view starts from Navarra, a territory subject to a significant repression in the months and years immediately following the 1936 military coup, and dialogues with other international experiences. The paper also aims to reflect on the opportunity of these new technological developments, bearing in mind the objective of recognition and reparation of the victims, as well as the visibility and preservation of their memory in a post-conflict society such as Navarre, but can be extrapolated to other geographic and chronological contexts.
\end{abstract}

KEYWORDS: Historical Memory, Sites of Memory, Digital Mediation, Digital Humanities.

* Correspondencia a: Álvaro Baraibar. Universidad Pública de Navarra. Departamento de Ciencias Humanas y de la Educación. Campus Arrosadia. Edifico los Acebos. 31006 Pamplona-Iruña (Navarra-Spain) - alvaro.baraibar@unavarra. es - https://orcid.org/0000-0001-5892-2604

Cómo citar: Baraibar, Álvaro (2021). "Mediación digital y memoria histórica: una mirada desde Navarra»; Historia Contemporánea, 65, 275-297. (https://doi.org/10.1387/hc.21092).

Recibido: 16 septiembre, 2019; aceptado: 18 marzo, 2020.

ISSN 1130-2402 - eISSN 2340-0277 / (C) 2020 UPV/EHU

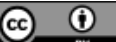


Álvaro Baraibar

\section{Consideraciones previas}

Si nos acercamos al debate historiográfico y al propio debate público, es evidente el especial interés que en los últimos tiempos ha suscitado la memoria histórica en España al hilo de las reivindicaciones de las familias de las víctimas y de las asociaciones memorialistas. Algo similar está ocurriendo también si nos asomamos al debate que se ha suscitado sobre el papel de lo digital, de la mediación digital, en el marco del trabajo en torno a las Humanidades. Sería, por tanto, ingenuo creer, y poco riguroso pretender, que el acercamiento que aquí se plantea a la mediación digital de la memoria histórica es totalmente novedoso ${ }^{1}$. Especialistas en Humanidades Digitales como Paul Spence ya se preocuparon hace un tiempo por este tema ${ }^{2}$. Del mismo modo, no son pocas las aportaciones que se han llevado a cabo a la intersección de estas dos categorías desde las diferentes disciplinas que engloban las Ciencias de la Memoria ${ }^{3}$.

En definitiva, el trabajo en torno a la memoria posibilita aproximaciones muy variadas, tanto por los diferentes ámbitos de conocimiento desde los que se analiza, como por los múltiples contextos geográficos y cronológicos de sociedades post-conflicto que se ven interpelados en estos estudios. Dentro de ese amplio abanico de posibilidades, el presente trabajo quiere acercarse al análisis del papel que desempeña, o podría ofrecer, la mediación digital en la implementación de iniciativas, tanto institucionales como privadas, que busquen el reconocimiento y la reparación de las víctimas y que, en esa labor, se enfrentan a la necesidad de dar respuesta a la gestión del patrimonio de la memoria en sus diferentes expresiones.

1 Ver Eiroa, 2018. Matilde Eiroa ha dirigido el proyecto «Historia y Memoria Histórica online (HISMEDI)» que analiza y recopila la presencia de materiales sobre la Guerra Civil y el franquismo en Internet: http://evi.linhd.uned.es/projects/hismedi/om/inicio [consultado el 10 de marzo de 2020]. Más modesta es otra iniciativa, «Vidas contadas» (http:// vidascontadas.org) [consultado el 10 de marzo de 2020], que reúne algunos materiales digitales relacionados con la memoria histórica en España.

${ }^{2}$ Spence, 2018. Ver también, entre otras, aproximaciones de temática más acotada como las de Eiroa, 2014; o Bocanegra y Toscano, 2016, sobre la presencia de la Guerra Civil en los en los medios de comunicación on line y sobre el exilio republicano, respectivamente.

${ }^{3}$ Una lectura de las páginas del extenso programa del Congreso de la Memory Studies Association, celebrado en Madrid entre el 25 y el 28 del mes de junio de 2019 da buena muestra de ello: https://www.memorystudiesassociation.org/madrid-conference-2019-infohome/ [consultado el 10 de marzo de 2020]. 
La mirada que aquí propongo se proyecta desde una realidad concreta, la de Navarra, una región que se vio fuertemente afectada por la represión de la sociedad civil por parte de las fuerzas sublevadas en 1936. En Navarra no hubo frente militar en ningún momento de la guerra. Aun así, más de 3000 personas fueron asesinadas y enterradas en fosas comunes clandestinas a consecuencia de la violencia desatada en la retaguardia ${ }^{4}$. El trabajo parte de este contexto geográfico, cronológico y victimológico, pero desde la consideración de que algunas de las conclusiones a las que se pueda llegar sobre el papel de la mediación digital podrán ser extrapolables a otros espacios y tiempos. Algunos de ellos, sin ninguna pretensión de exhaustividad, aparecerán en las próximas páginas por la relevancia que tienen en el desarrollo del estudio.

$\mathrm{El}$ artículo caminará, en primer lugar, atendiendo específicamente al patrimonio de la memoria de las víctimas de la represión franquista. En ese contexto se analizará el papel de los lugares de la memoria en el recuerdo del pasado, así como la función que la mediación digital puede desempeñar en el reconocimiento y la reparación de las víctimas y a la hora de «reactivar "recuerdos" comunitarios olvidados y de esa forma consolidar culturas del pasado en red ${ }^{5}$. En segundo lugar, el trabajo se acercará a la gestión de los vestigios del terror, con el objetivo de mostrar de qué manera lo digital puede aportar ideas acerca de cómo tratar la simbología y nomenclatura de la dictadura franquista. Y en tercer lugar, se mostrarán algunas posibilidades de tratamiento digital de los testimonios de las víctimas y sus familias, iniciativas que tienen el ánimo de crear un corpus de relatos que garanticen la memoria de lo ocurrido y eviten el olvido social e institucional de quienes sufrieron las consecuencias de la violencia.

\section{El patrimonio de la memoria de las víctimas}

\subsection{Lugares de memoria/Lugares de conciencia}

La idea de patrimonio es abierta, multidisciplinar y está en continuo proceso de transformación y crecimiento. Elementos como el patrimo-

4 Altafaylla, 2018; Gobierno de Navarra, 2019. Ver también la base de datos del Fondo Documental de la Memoria Histórica en Navarra: https://memoria-oroimena.unavarra.es [consultado el 10 de marzo de 2020].

5 Noiret, 2018, p. 113, quien cita a la International Coalition of Sites of Conscience, a la que nos referiremos más adelante. 
nio inmaterial, el patrimonio industrial o el paisaje cultural han ido enriqueciendo el concepto de patrimonio y ensanchando, al mismo tiempo, el abanico de posibilidades didácticas a nuestra disposición a la hora de acercar al conjunto de la sociedad nuestra herencia y comprensión del pasado, nuestra cultura y nuestras propias señas de identidad.

Una de las últimas miradas que ha incorporado el concepto de patrimonio ha sido la memorialista. Siguiendo el camino que transitaron lo inmaterial o esa suerte de interacción entre lo natural y lo humano que es el paisaje cultural, lo memorial suma al patrimonio la idea de lugares de memoria que acuñara hace ya más de veinte años el historiador francés Pierre Nora ${ }^{6}$. Los lugares de memoria son espacios de una especial significación como símbolos de momentos y de acontecimientos que se dieron en el pasado pero que cobran relevancia en nuestro presente y se proyectan también hacia nuestro futuro. El valor de estos lugares no está necesariamente, o puede no estar, tanto en lo patrimonial, entendido en un sentido tradicional, sino en la relevancia de lo ocurrido en ese lugar en un pasado más o menos remoto. Es el recuerdo de unos determinados acontecimientos lo que hace que el lugar en el que se produjeron cobre la condición de lugar de memoria, de lugar a través del que reconocer, recordar y difundir un suceso de nuestro pasado.

Más allá del ámbito académico y del significado de los lugares de memoria estudiados por Nora, el trabajo de un buen número de iniciativas a nivel internacional y el desarrollo, posteriormente, de algunas políticas públicas de memoria han avanzado en una mayor concreción de lo que son o pueden ser los lugares de la memoria vinculados a hechos traumáticos del pasado en países con realidades históricas muy distintas. A estos lugares se les ha llamado también «sitios de conciencia» (Sites of Conscience) y desde 1999 se han constituido en una red internacional que ha ido creciendo de manera significativa: la International Coalition of Sites of Conscience (ICSC) ${ }^{7}$.

Esta mirada me parece especialmente relevante por varias razones. Desde esta perspectiva, los lugares de la memoria incorporan a nuestro patrimonio el recuerdo del pasado y, permiten avanzar, a través de distintas iniciativas, en la voluntad de reconocimiento y reparación individual, familiar y social de hechos traumáticos de nuestra historia desde el

\footnotetext{
6 Nora, 1997.

7 https://www.sitesofconscience.org/en/home/ [consultado el 10 de marzo de 2020].
} 
compromiso con la convivencia y los Derechos Humanos. Los lugares de la memoria - desde esta dimensión como lugares de la conciencia - serían, por tanto, espacios vinculados a hechos violentos del pasado, al terror, pero que, a través de un trabajo pedagógico y de visibilización de los valores de la democracia, el respeto y los Derechos Humanos, se pueden convertir en espacios de convivencia y de fomento de una cultura de $\mathrm{paz}^{8}$. Se trata, en definitiva, de espacios que trascienden el valor como símbolo de la identidad de una comunidad o nación que Nora reconocía a los lugares de memoria y les confiere un significado global, humano, desde una mirada crítica al pasado, a lo ocurrido en nuestra historia lejana y reciente.

A finales del año 2018, el Parlamento de Navarra aprobó la Ley Foral 29/2018, de 26 de diciembre, de Lugares de la Memoria Histórica de Navarra, muy expresiva al respecto de esta perspectiva sobre los lugares de la memoria (o de la conciencia). Según dice el texto, un lugar de la memoria histórica es

«aquel espacio físico ubicado en Navarra y declarado e inscrito como tal, que sea de interés para la Comunidad Foral como patrimonio histórico de la memoria por haberse desarrollado en él hechos de singular relevancia vinculados con la represión y violencia ejercidas sobre la población como consecuencia del golpe militar de 1936 y la subsiguiente represión franquista» ${ }^{9}$.

También podrá ser lugar de memoria un espacio que, «aun no estando vinculado directa e históricamente a aquellos acontecimientos, en fechas posteriores, las familias de las víctimas, las asociaciones memorialistas, las instituciones y las administraciones públicas hayan erigido en recuerdo, reconocimiento y reparación de las víctimas de aquella represión» ${ }^{10}$. El objetivo del texto legislativo es «proteger estos lugares de memoria, regular su señalización y conservación, y promover su conocimiento, con el ánimo de convertir aquellos lugares de terror en espacios de recuerdo y transmisión de valores de paz y convivencia» ${ }^{11}$.

${ }^{8}$ Esta idea es la que inspira, por ejemplo, el mapa interactivo \#MemoriasSituadas del Centro Internacional para la Promoción de los Derechos Humanos de la UNESCO: https:// www.cipdh.gob.ar/memorias-situadas/ [consultado el 25 de noviembre de 2020].

9 Ley Foral 29/2018, art. 2. Sobre el desarrollo de la Ley, ver Gastón y Layana, 2020.

10 Ley Foral 29/2018, art. 2.

11 Ley Foral 29/2018, Preámbulo. 
La Ley navarra camina en la dirección que, unos meses antes, trazaba la propia UNESCO. El 31 de enero de 2018, este organismo internacional hizo público un documento titulado Interpretation of Sites of Memory ${ }^{12}$, resultado del trabajo de la International Coalition of Sites of Conscience a la que ya me he referido. La iniciativa partía de un encargo del Centro del Patrimonio Mundial de la UNESCO a partir de la decisión adoptada por el Comité y de las recomendaciones del Congreso Internacional sobre Interpretación del Patrimonio Mundial celebrado en Seúl (Corea) en 2016. Tal y como recoge dicho documento,

«Sites of Memory for the purposes of this report are places which are vested with historical, social or cultural significance because of what has happened there in the past. [...] In a Site of Memory, the associative values can be of greater importance than the material ones and can convey a variety of meanings, even though the material remains can be vital in understanding the associative values» ${ }^{13}$.

El texto desarrolla, además, con precisión los matices que diferencian, desde su punto de vista, los lugares de la conciencia y los lugares de la memoria, apuntando, como veremos, en la misma dirección que la ley navarra. Un lugar de la conciencia reúne cuatro características fundamentales: interpreta la historia a través de lo acontecido en un espacio determinado; estimula la reflexión y el diálogo social sobre aspectos de alto valor actual; se abre al debate público y la participación; y promueve los valores de la justicia y los Derechos Humanos ${ }^{14}$. Por ello, «all Sites of Conscience are Sites of Memory, but many Sites of Memory are not Sites of Conscience» ${ }^{15}$.

Desde esta perspectiva, mientras los lugares de la memoria de Nora conectan el presente con aspectos identitarios específicos de la comunidad que se buscan en el pasado, los lugares de la conciencia bucean en el pasado para abrir el debate acerca de valores universales propios de nuestro presente y proyectan su mirada hacia el futuro: «A Site of Conscience is a Site of Memory, but one that actively confronts the history of what happened at that site and spurs visitors to reflect and act on the history's contemporary

\footnotetext{
12 International Coalition of Sites of Conscience, 2018.

13 International Coalition of Sites of Conscience, 2018, p. 13.

14 International Coalition of Sites of Conscience, 2018, pp. 14-15.

15 International Coalition of Sites of Conscience, 2018, p. 15.
} 
implications» ${ }^{16}$. En definitiva, «while Sites of Conscience and Sites of Memory are both intimately connected with the past, Sites of Conscience are distinct in their steadfast commitment to the future. Their exhibition content, public programming and mission are resolutely forward-thinking» ${ }^{17}$.

\subsection{La mediación digital del patrimonio de la memoria de las víctimas}

Textos como Interpretation of Sites of Memory o como la Ley Foral de Lugares de Memoria Histórica de Navarra tienen, además de lo memorial, otro aspecto en común que comienza a estar presente en este tipo de documentos: ambos incorporan una mención explícita a las oportunidades que lo digital ofrece a la hora de trabajar en torno al patrimonio de la memoria, a los lugares de la memoria ${ }^{18}$. Las referencias a lo digital en este tipo de «literatura gris», incluso en textos legislativos como la Ley navarra, muestra, a mi modo de ver, cómo las Humanidades Digitales - explícita o implícitamente, haciendo referencia al término o no- han trascendido el ámbito académico y han permeado otros espacios como el de la gestión de la memoria, tanto desde instituciones públicas como privadas.

El documento de la UNESCO alude al uso de varias técnicas digitales cuando trata de enumerar algunos de los medios con los que se puede trabajar la preservación y divulgación de los lugares de la memoria. Entre estas técnicas cita explícitamente la realidad virtual, aumentada, mixta o inmersiva, las apps para móviles, los new media para la grabación, preservación y divulgación de los testimonios o los museos virtuales ${ }^{19}$. Del mismo modo, la Ley navarra, en su artículo 17, que regula las actividades de divulgación en torno a los lugares de la memoria histórica, contempla que la administración «favorecerá el acceso a la memoria histórica a través de Tecnologías de la Información y la Comunicación y suministrará mediante ellas toda la información necesaria relativa a los lugares de la memoria histórica de Navarra» ${ }^{20}$.

16 International Coalition of Sites of Conscience, 2018, p. 25.

17 International Coalition of Sites of Conscience, 2018, p. 25.

18 Para otro caso interesante de referencias a lo digital en documentos oficiales que caminan en este mismo sentido de los lugares de la memoria ver el dictamen del Comité Económico y Social Europeo (2019) sobre «La Senda Paloma de la Paz (White Dove Way)».

19 International Coalition of Sites of Conscience, 2018, p. 20.

${ }^{20}$ Ley Foral 29/2018, art. 17. 
¿Cuáles han sido las aproximaciones digitales al patrimonio de la memoria histórica? En primer lugar, me referiré al Mapa de Lugares de la Memoria Histórica, regulado por la Ley Foral 29/2018 que explicita cómo el departamento del Gobierno de Navarra «competente en materia de memoria histórica elaborará y mantendrá actualizado un mapa digital, de acceso público a la ciudadanía, donde se ubiquen geográficamente los lugares de la memoria histórica de Navarra y se informe de lo que allí ocurrió» ${ }^{21}$.

El Mapa de Lugares de la Memoria Histórica se presentó el 3 de julio de 2019. Se trata de un Sistema de Información Geográfica (SIG) que, a partir de la georreferenciación de los datos introducidos en una base, es capaz de mostrar la información sobre un mapa ${ }^{22}$. El Mapa de Lugares se ha implementado sobre la base del Mapa de Fosas que el Gobierno de Navarra desarrolló con la empresa pública TRACASA en respuesta a la Ley 52/2007 de memoria histórica. Los datos que muestra el mapa de fosas y mapa de Lugares de la Memoria forman parte del Sistema de Información Territorial de Navarra (SITNA) y que, en consecuencia, todos los datos son accesibles también a través de la Infraestructura de Datos Espaciales de Navarra (IDENA) y de la plataforma de datos abiertos del Gobierno de Navarra. Es relevante destacar este aspecto porque la puesta a disposición en abierto de otros proyectos de los datos generados en las investigaciones es un aspecto definitorio de las Humanidades Digitales y que cuestiona de raíz calificativos utilizados por Aibar Puentes, quien considera esta disciplina como un ejemplo de la «transformación neoliberal de la ciencia» ${ }^{23}$.

En el caso del mapa de Lugares de la Memoria Histórica de Navarra se ha implementado una interfaz muy sencilla y fácil de manejar por usuarios que no precisan de ningún conocimiento específico, teniendo en cuenta que, además de un objetivo documentalista, el Mapa tiene también y específicamente un sentido reparador para personas que, en muchos casos, tienen hoy, pasados ya más de 80 años de aquellos acontecimientos, una edad muy avanzada ${ }^{24}$.

${ }^{21}$ Ley Foral 29/2018, art. 16.

22 La base de datos que se está utilizando es PostgreSQL (extensión espacial PostGIS). Para un extenso trabajo sobre la aplicación de las posibilidades de las Sistemas de Información Geográfica en el marco de las Humanidades Digitales, ver Gámir, 2019.

${ }^{23}$ Aibar Puentes, 2018.

24 Sobre la voluntad reparadora de este mapa, resulta especialmente relevante la conexión llevada a cabo con la base de datos del Fondo Documental de la Memoria Histórica y anunciada por el Instituto Navarro de la Memoria el 12 de agosto de 2020: https://www. navarra.es/es/noticias/2020/08/12/el-gobierno-conecta-el-mapa-de-fosas-de-navarra-conel-fondo-documental-de-la-memoria-historica-de-la-comunidad-foral. Es interesante ver 
Cada uno de los Lugares de Memoria está ubicado en sus coordenadas cartográficas y cuenta con una breve ficha informativa, en la que se recogen su número identificador en el Registro de Lugares de Memoria, una reseña sobre su significación memorialista y las referencias legales sobre su declaración como Lugar de la Memoria Histórica de Navarra. La ficha se completa con material fotográfico o audiovisual sobre el lugar. El Mapa se puede consultar de forma independiente o, si el usuario lo desea, en combinación con el resto de referencias cartográficas sobre fosas y espacios de memoria, recogidos en distintas capas del $\mathrm{SIG}^{25}$.

Considero que es importante resaltar el valor no solo informativo de estas aplicaciones, sino también el componente reparador del mapa en sí mismo: reparador para las familias de las víctimas del golpe militar que ven cómo la máxima institución de su Comunidad establece el relato de lo que le ocurrió a su ser querido (cuando ha sido posible la localización e identificación de las víctimas), y reparador también para las personas que quieran aportar una información que permita ubicar la localización de una fosa común. Por ello, en lo relativo al mapa de fosas, la incorporación de nuevas informaciones está abierta a la ciudadanía.

En el trabajo en torno a los lugares de la memoria, si el objetivo es el de reconocer y reparar a las víctimas, es imprescindible que la labor institucional se traslade al conjunto de la sociedad. Por ello, tal y como ha afirmado Noiret al hablar sobre historia pública digital y redes sociales, «compartir un conocimiento participativo crea conciencia pública sobre esos pasados en nuestro presente» ${ }^{26}$. A pesar de estar de acuerdo con esta afirmación, creo que en el particular que nos ocupa hay que tener en cuenta una cuestión más. Si bien es cierto que la información que se muestra en Internet multiplica de forma exponencial el público potencial, no lo es menos que el usuario de estas aplicaciones tiene que hacer el esfuerzo de acceder a ellas.

también el Mapa de Fosas de Andalucía, en el que se ha tenido en cuenta, partiendo de la realidad específica de aquella región, la posibilidad de que algunas fosas hayan sido dignificadas: https:/www.juntadeandalucia.es/organismos/culturaypatrimoniohistorico/areas/ memoria-democratica/fosas/mapas-fosas.html [consultado el 10 de marzo de 2020]. Ver, al respecto, Barrera Becerra, Giráldez Díaz y Melero Vargas, 2020.

25 Ver http://fosas.navarra.es [consultado el 10 de marzo de 2020]. El mapa permite la búsqueda por varios criterios y el usuario puede marcar más o menos capas, de modo que se active la información relativa a los lugares de la memoria o también, al mismo tiempo, a las fosas comunes, tanto intervenidas como no, con información, en el primero de los casos, sobre las personas recuperadas en cada una de ellas.

26 Noiret, 2018, p. 114. 
Álvaro Baraibar

Ese acto de voluntad lo llevará a cabo, evidentemente, el sector de la sociedad que por una motivación natural o profesional esté interesado en la materia. En un marco académico, un enfoque de este tipo estaría plenamente justificado. Sin embargo, en el contexto de la gestión de las políticas públicas de la memoria no sería así y podría incluso ser entendido como un intento de velar digitalmente, de ocultar, en cierta medida, la memoria en un contexto digital. Este es un aspecto que debe estar presente en el desarrollo institucional de estas aplicaciones y en la posibilidad -o necesidad - de acompañarlas con actuaciones en el espacio físico, por medio de paneles informativos, de forma que ambos entornos, el digital y el físico, sean complementarios ${ }^{27}$.

En el marco de los Lugares de la Memoria, concretamente de los espacios memoriales construidos para el recuerdo de las víctimas, uno de los elementos más relevantes y presentes en distintos lugares del mundo con realidades post-conflicto es, sin duda, el muro de los desaparecidos, el muro de las víctimas. El componente reparador de este tipo de espacios conmemorativos es especialmente significativo porque, entre otros aspectos, incorpora un reconocimiento individual y colectivo a quienes sufrieron los efectos de la violencia. Los muros de las víctimas o de los desaparecidos reconocen y reparan a cada una de las víctimas y las sitúan, además, en el recuerdo del grupo que padeció la misma injusticia.

Muros de este tipo los podemos encontrar en Buenos Aires (el Parque de la Memoria-Monumento a las Víctimas del Terrorismo de Estado) ${ }^{28}$, en Santiago de Chile (tanto el Memorial del Detenido Desaparecido y del Ejecutado Político en el Cementerio General como la Villa Grimaldi) ${ }^{29}$, en Montevideo, Uruguay (Memorial en Recordación de los Detenidos y

27 Desde esta perspectiva considero especialmente interesantes iniciativas como la desarrollada por el Ayuntamiento de Sartaguda (Navarra), localidad trágicamente afectada por la violencia represiva del 36 , que ha marcado un recorrido de la memoria por el municipio con paneles informativos que complementan la información por medio de materiales en Internet a través de códigos QR. Ver «Paseo por la Memoria/Memoriaren Ibilbidea»: http://paseoporlamemoria.sartaguda.net/index.html [consultado el 10 de marzo de 2020]. Quiero agradecer a Daniel Palacios González las conversaciones mantenidas a este respecto en el marco de su trabajo sobre las prácticas monumentales en torno a las fosas comunes en España («Monumental Practices on Spanish Civil War Mass Graves») y que me han sido de gran ayuda, especialmente en lo relativo a la idea del «velo digital».

${ }^{28} \mathrm{http}: / /$ parquedelamemoria.org.ar [consultado el 10 de marzo de 2020].

${ }^{29}$ El muro de los nombres, en Villa Grimaldi http://villagrimaldi.cl/parque-por-la-paz/ muro-de-los-nombres/ [consultado el 10 de marzo de 2020] y el Memorial del Detenido Desaparecido y del Ejecutado Político en el Cementerio General de Santiago. A diferencia de 
Desaparecidos $)^{30}$. También en Sartaguda, Navarra, se ha erigido un Parque de la Memoria destinado al recuerdo de los más de 3000 desaparecidos forzados a consecuencia de la violencia del franquismo.

En este sentido, aunque nos aleje de la memoria del franquismo, la iniciativa Fushat Amal (Líbano) - que podríamos traducir como «Espacio de esperanza»- es, a mi modo de ver, especialmente interesante como aproximación a la memoria desde las Humanidades Digitales ${ }^{31}$. La web tiene una doble dimensión. En primer lugar, como herramienta fundamental para recopilar y dar a conocer la memoria de las 17000 personas que se estima que desaparecieron en el conflicto del Líbano, entre 1975 y 1990. Se trata de una herramienta dirigida a la construcción de un censo de víctimas, concretamente de personas desaparecidas, un elemento prioritario desde la perspectiva de los Derechos Humanos en el trabajo en sociedades post-conflicto. Es, en definitiva, una base de datos, un tipo de proyecto que, de alguna manera, ha perdido protagonismo en el ámbito de las Humanidades Digitales, más interesadas en impulsar iniciativas con un alto componente de innovación tecnológica.

Pero el caso de Fushat Amal tiene una segunda dimensión. Más allá de ser una base de datos, la web es también, en sí misma, un espacio memorial dedicado al recuerdo de las personas desaparecidas en el conflicto del Líbano. La idea de este espacio memorial virtual se inspira, como decía, en los muros de las personas desaparecidas; es, de hecho, un muro digital de las víctimas. El propio diseño de la web ha querido tener presente esta idea. Por ello, Fushat Amal tiene un apartado dedicado a dignificar su memoria ofreciendo la información existente de cada una de ellas, abierta, además, a las aportaciones de cualquier persona que tenga información al respecto.

Los muros de las víctimas, especialmente si se trata de personas desaparecidas, son el resultado de proyectos de investigación promovidos en muchos casos con grandes dificultades por la ausencia de documentación que permita constatar dichas victimaciones. Se trata de bases de datos elaboradas con un enorme esfuerzo para tratar de determinar el censo de personas desaparecidas y de víctimas de un proceso de vulneraciones de De-

la Villa Grimaldi, integrada en la red de Sites of Conscience y recogida por Interpretation of Sites of Memory, no he encontrado una página oficial, institucional, para este muro memorial.

${ }^{30} \mathrm{http}: / /$ montevideo gub.uy/areas-tematicas/ciudad-y-urbanismo/patrimonio/memorial-en-recordacion-de-los-detenidos-desaparecidos [consultado el 10 de marzo de 2020].

31 https://www.fushatamal.org [consultado el 10 de marzo de 2020]. 
Álvaro Baraibar

rechos Humanos. Así ocurre en los países mencionados ${ }^{32}$, y así ocurre también en España a iniciativa de diferentes Comunidades Autónomas, de proyectos universitarios o de asociaciones y colectivos ${ }^{33}$. Bases de datos con un mayor o menor desarrollo tecnológico, pero sin las que sería imposible la elaboración de censos o el análisis de los patrones de la violencia represiva en regímenes dictatoriales o en contextos de vulneración de los Derechos Humanos.

Antes me he referido a la necesidad de trabajar en torno a los lugares de la memoria buscando la complementariedad entre espacio físico y espacio virtual. En el caso de los muros de las víctimas, donde el elemento memorial alude de manera explícita a las personas, a sus nombres, esta idea cobra nuevas dimensiones. En casos como este ocurre que hay familias que, por diferentes motivos, no quieren que el nombre de su ser querido aparezca en el muro. En estos casos la mediación digital puede ser la solución, justo al contrario de lo expuesto en el caso anterior. Ese velo digital puede hacer que la memoria de algunos nombres esté presente virtualmente, sin estarlo de forma física ${ }^{34}$.

32 Sin voluntad de exhaustividad, que excedería el objetivo y ámbito de este trabajo, es interesante dirigir la mirada también a otras experiencias como son, por ejemplo, los casos de Argentina (http://basededatos.parquedelamemoria.org.ar) [consultado el 10 de marzo de 2020] o Chile (http://interactivos.museodelamemoria.cl/victimas/) [consultado el $10 \mathrm{de}$ marzo de 2020].

${ }^{33}$ En Navarra, el Proyecto del «Fondo Documental de la Memoria Histórica de Navarra» del equipo Recuperando Memoria de la Universidad Pública de Navarra dirigido por Fernando Mendiola: https://memoria-oroimena.unavarra.es [consultado el 10 de marzo de 2020]. En Galicia, el Proyecto «Nomes e Voces» de las Universidades de Galicia, dirigido por Lourenzo Fernández Prieto: http://vitimas.nomesevoces.net [consultado el 10 de marzo de 2020]. Y en Andalucía, Extremadura y Norte de África el Proyecto «Todos los nombres», impulsado por la CGT: http://www.todoslosnombres.org [consultado el 10 de marzo de 2020]. También es interesante en este sentido la base de datos del proyecto «SUBTIERRO: Exhumaciones de fosas comunes y derechos humanos en perspectiva histórica, transnacional y comparada», dirigido por Francisco Ferrándiz (https://politicasdelamemoria.org); ver Ferrándiz Martín, Salas Tovar y De Kerangat, 2017. Y desde un punto de vista archivístico, cabe destacar la iniciativa «Víctimas de la Guerra Civil y Represaliados del Franquismo», impulsada por el Ministerio de Cultura y Deporte en PARES (http://pares.mcu.es/victimasGCFPortal/staticContent.form?viewName=presentacion) [consultado el 10 de marzo de 2020].

${ }^{34}$ Este aspecto es especialmente importante en contextos en los que se producen agresiones a la memoria de las víctimas y requiere de una reflexión pausada para evitar segundas revictimaciones. Las agresiones a las víctimas tienen lugar no solo en un contexto físico, sino también digital; tal vez de manera especialmente intensa por medio de redes sociales como Twitter. En este sentido, creo que la mirada de Paniagua Santamaría (2018) sobre el efecto de esta red social en el trabajo por la memoria es excesivamente positiva. 
Un caso reciente, en el que intervienen otros factores pero que es igualmente ilustrativo, es el que hemos podido ver en Madrid en torno al proyecto de construcción de un espacio memorial a las víctimas de la represión en el cementerio de La Almudena ${ }^{35}$. En este caso, la solución propuesta por el Comisionado de la Memoria Histórica del Ayuntamiento de Madrid fue la instalación de dos placas sin nombres, pero accesibles a través de un código $\mathrm{QR}^{36}$.

\section{La mediación digital en la gestión del patrimonio del terror ${ }^{37}$}

Como hemos podido comprobar hasta ahora, el patrimonio de la memoria, cuando se trata de la memoria de periodos de conflicto y vulneraciones de Derechos Humanos, es, habitualmente, un patrimonio incómodo, utilizando el concepto acuñado por Prats ${ }^{38}$. Hernàndez i Martí, siguiendo la idea de sombra de Jung, ha afirmado que existe una «dificultad inherente a la gestión de la patrimonialización de la sombra o 'memoria oscura' ${ }^{39}$. Pero esta idea se acentúa más aún cuando esa gestión es institucional y no se construye al modo de una contramemoria frente al discurso dominante sino de una memoria erigida sobre los pilares de los Derechos Humanos, de la ética y de una mirada crítica al pasado ${ }^{40}$. Llegados a este punto, conviene recordar las palabras de Adorno: «dejar hablar al sufrimiento es la condición de toda verdad» ${ }^{41}$.

35 García-Funes, 2020.

36 Ver https://elpais.com/ccaa/2018/04/25/madrid/1524692256_260856.html [consultado el 10 de marzo de 2020].

37 Soy consciente de las importantes aportaciones que, en la preservación digital, la visibilización del patrimonio de la memoria y la investigación sobre la cultura digital se han llevado a cabo desde Museos, Archivos, Bibliotecas y Galerías. Especialmente interesantes son las experiencias de creación colaborativa de materiales digitales en abierto que desde hace años están llevando a cabo los grupos GLAM Creative Commons (https://wiki.creativecommons.org/wiki/GLAM) y, con contenidos directamente relacionados con la memoria, de Wikimedia (https://outreach.wikimedia.org/wiki/GLAM) [consultado el 10 de marzo de 2020]. La bibliografía al respeto es ingente y requeriría un espacio y un esfuerzo que supera el marco del presente trabajo. En cualquier caso, un interesante portal es Open Glam (https://medium.com/open-glam) [consultado el 10 de marzo de 2020].

38 Prats, 1997, p. 35. Posteriormente el concepto ha tenido éxito y ha sido utilizado en varios trabajos, algunos, como el de Sánchez Carretero (2013) o el de Bianchini (2015), explícitamente relacionados con el tema que nos ocupa.

39 Hernàndez i Martí, 2010.

40 Mate, 2016. Ver también Traverso, 2007.

41 Adorno, 1992, p. 27. Sobre este particular, ver también Mate, 2013, entre otros trabajos. 
Hasta aquí hemos visto casos de cómo se ha abordado la memoria de las víctimas, de las personas desaparecidas o que han sufrido la represión del franquismo y otros regímenes dictatoriales. Sin embargo, las Administraciones, además de afrontar esta memoria, se enfrentan también a la necesidad de gestionar el patrimonio cultural que representan los símbolos y elementos de exaltación de esos mismos regímenes dictatoriales. En definitiva, ¿qué hacer con los símbolos de exaltación de la violencia del franquismo o de quienes la ejercieron? Es aquí cuando el grado de incomodidad se acentúa.

En la misma línea de la aplicación de las posibilidades de los Sistemas de Información Geográfica al patrimonio de la memoria, me gustaría mencionar el mapa interactivo desarrollado para mostrar el proceso de retirada de simbología franquista llevado a cabo en Navarra entre 2015 y 2019. En esta ocasión la forma elegida fue la de una pequeña exposición instalada en un container en el centro de Pamplona, en junio de 2019, bajo el título Deconstruir el franquismo. Democratización del espacio público. Retirada de simbología. La exposición fue comisariada por Jordi Guixé, director del EUROM (Observatorio Europeo de Memorias), y Núria Ricart, profesora de Bellas Artes de la Universidad de Barcelona. La muestra combinaba una selección de placas retiradas, paneles informativos y una pantalla táctil que mostraba una base de datos con la información georreferenciada de los símbolos retirados, su ubicación y significado, además de imágenes de todos ellos.

El proyecto buscaba explicar a la ciudadanía las razones de la retirada de esta simbología, su significado de exaltación de la violencia - incompatible con la democracia - y, de un modo especial, la ofensa y revictimación que representan para las personas que sufrieron la injusticia de la violencia y para sus familias.

¿Musealizar los símbolos del franquismo responde a estos objetivos, podríamos preguntarnos? Al llevar los símbolos del franquismo de nuestras calles y plazas a una exposición (permanente o temporal) estamos eliminando su sentido de exaltación de la violencia, de la dictadura, del valor que el discurso oficial de la dictadura otorgó a la violencia y a la guerra. Su retirada del espacio público anula la función para la que fueron ideados e instalados, evitando la pervivencia de elementos dictatoriales en plena democracia. Pero al mismo tiempo, al exponer esos símbolos a la ciudadanía, en el marco de un discurso de profundización democrática y deslegitimación de la violencia, evitamos la desmemoria, el olvido que posibilita la manipulación de la historia y que impide el establecimiento de garantías de no repe- 
tición. Como afirma Escribano Gonzálvez en un trabajo sobre la musealización de la memoria de las víctimas del franquismo:

La exposición de la barbarie y la guerra queda justificada en las nuevas museografías por la necesidad de preservar y conservar espacios patrimoniales que fueron escenario de las tragedias, así como archivos, objetos y documentos resultado de estas herencias incómodas como herramientas de legitimación y prevención, que puedan transmitir lo ocurrido y ofrezcan al visitante una visión crítica, didáctica e incluso emocional ${ }^{42}$.

Desde una perspectiva distinta, Hernàndez i Martí parece respaldar también esta idea cuando afirma que uno de los principales retos de la patrimonialización de la cultura y de su gestión es precisamente

la progresiva incorporación, asimilación, integración y activación de la «memoria oscura», de la sombra patrimonial, del extenso territorio que hemos ido dejando abandonado, sumergido, oculto, consciente o inconscientemente, al creer que su negatividad sería de crudo impacto en el presente ${ }^{43}$.

En este sentido, la muestra Deconstruir el franquismo está basada en la elaboración de una base de datos que muestra sobre un mapa interactivo la simbología franquista que ha sido retirada. La base de datos recoge la tipología simbólica, una imagen del objeto retirado, así como las coordenadas de su ubicación geográfica para su posterior mapeado. El visitante, por medio de una pantalla táctil interactiva, puede lanzar una consulta tanto por municipio como por tipología simbólica o, incluso, por una combinación de ambas, activando y desactivando cada opción según sus intereses ${ }^{44}$.

${ }^{42}$ Escribano Gonzálvez, 2018, p. 271. De Bernardi (Universidad de Bolonia) llevó a cabo una interesante reflexión sobre la musealización del fascismo, en este caso en Italia, en su intervención en la I Jornada Internacional «Simbología dictatorial, arte y espacio público», Pamplona, 1 de junio de 2017. Su conferencia, titulada «La Casa del Fascio de Predappio», puede verse en http://www.navarra.es/home_es/especial/Agendamemoriahistorica/1junio.htm [consultado 10 de marzo de 2020]. Ver también Pasetti, 2018.

43 Hernàndez i Martí, 2010, p. 635.

${ }^{44}$ Un vídeo en el que se muestra las ideas clave de la exposición puede verse en https:// www.youtube.com/watch?v=D9QJAiQFaZ0 [consultado el 10 de marzo de 2020]. Esta iniciativa fue una adaptación de la idea original creada por los propios Guixé y Ricart para una exposición itinerante impulsada por el Comisionado de Programas de Memoria del Ayuntamiento de Barcelona e instalada en la antigua Cárcel de la Modelo en la primavera de 2019: http://ajuntament.barcelona.cat/programesmemoria/es/placa-memoria/deconstruir-el-franquismo-simbolos-de-la-dictadura-en-barcelona/ [consultado el 10 de marzo de 2020]. 


\section{Testimonios digitales de las víctimas y sus familias}

El patrimonio de la memoria histórica es, en cualquier caso, más extenso y variado que el relacionado con los lugares de la memoria y la simbología franquista. La propia memoria de lo ocurrido, así como la memoria de las víctimas y sus familiares, son aspectos clave para evitar la desmemoria, la injusticia del olvido. En todas estas cuestiones, la mediación de lo digital puede marcar una diferencia cualitativa a la hora de responder al derecho de las víctimas a la verdad, la justicia y la reparación. Como han afirmado Ferrándiz y Baer:

If the purpose of memory sites is to «stop the time, to block the work of forgetting (...) to immortalize death, to materialize the immaterial (...) in order to capture a maximum of meaning» (Nora, 1989, p. 19), we can plausibly assume that the audiovisual archive (photographs and video of the exhumation, testimonies, etc.) has become the genuine lieux de memorie in a digital memorial culture ${ }^{45}$.

Son varias las iniciativas que ya se han puesto en marcha para la preservación y divulgación de la memoria oral a través de Internet, aunque con resultados muy diferentes. Fenollosa Tejedor y Moncusí Ferre analizaron, en un trabajo de hace ya unos años, tres casos de patrimonialización de la memoria oral a través de Internet ${ }^{46}$. El más interesante de los tres era, sin lugar a duda, el Visual History Archive, de la USC Shoah Foundation ${ }^{47}$. El archivo nació para preservar la memoria de los supervivientes del Holocausto, aunque posteriormente ha incorporado también otras realidades, como el genocidio Tutsi, la masacre de Nanjing, el genocidio armenio, etc. Sus principales valores son, además del gran volumen de testimonios recogidos, la representación geográfica por medio de Google Maps de los topónimos mencionados en los testimonios y, sobre todo, la profundidad de marcado de los diferentes segmentos del material audiovisual por medio de un completo tesauro, aspecto este que enriquece enormemente las posibilidades de búsqueda ${ }^{48}$.

45 Ferrándiz y Baer, 2008, párrafo 35.

46 Fenollosa Tejedor y Moncusí Ferre, 2012.

47 Ver http://sfi.usc.edu/vha [consultado el 10 de marzo de 2020].

48 El tesauro desarrollado por la Fundación, uno de los primeros en este ámbito, se encuentra disponible públicamente en su web: https://sfi.usc.edu/content/keyword-thesaurus [consultado el 10 de marzo de 2020]. 
En España, en los últimos años, son varias las instituciones que han impulsado la creación de centros de documentación de la memoria ${ }^{49}$. El Memorial Democràtic de Cataluña (2007) fue el primero de ellos ${ }^{50}$. El Memorial es, probablemente, el organismo institucional que ha construido el centro de documentación más desarrollado en lo que a la recopilación de la memoria de las víctimas del franquismo se refiere, también en lo relativo a su testimonio. Otras instituciones como el Gobierno Vasco, a través de Gogora, Instituto de la Memoria, la Convivencia y los Derechos Humanos $^{51}$ o el Gobierno de Navarra, por medio del Instituto Navarro de la Memoria, están trabajando en la misma línea ${ }^{52}$.

El Banco de la Memoria del Memorial Democràtic ha sido desarrollado a partir de Dédalo, una plataforma abierta que se ideó para la gestión de archivos audiovisuales especializados en patrimonio inmaterial o historia oral ${ }^{53}$. Sobre la base de Dédalo se han construido varios proyectos en España, como El Museu de la Paraula o Maternidades robadas, sobre el caso de los conocidos como bebés robados, o en otros lugares, como Alemania, con el proyecto de Memorias de la ocupación en Grecia.

El Banco del Memorial integra distintas colecciones y soportes documentales: el banco audiovisual de testimonios, el censo de simbología franquista, la base de datos de ex-presos políticos y una biblioteca, entre otros. El interfaz permite lanzar búsquedas específicas en cada uno de ellos por separado o, de manera conjunta, en todos al mismo tiempo, algo especialmente interesante para quien, tanto en el marco de una investigación académica como privada - personal o familiar - pretenda documen-

49 Para una mirada complementaria a lo institucional, centrada en la producción cinematográfica y otras «contribuciones singulares» ver el capítulo «Imágenes del pasado en las plataformas digitales: historia, memoria y ficción» (Eiroa, 2018). La propia autora llama la atención en su trabajo sobre algunos riesgos de esa «intersección entre la ficción, el periodismo y la historia» y reivindica el papel del trabajo historiográfico (Eiroa, 2018, pp. 145-146).

50 El Preámbulo de la Ley 13/2007, de 31 de octubre, que creó el Memorial explica cómo el centro estaba llamado a ser un «instrumento con el que la Generalidad debe llevar a cabo políticas públicas destinadas a la recuperación de la memoria de las víctimas de la Guerra Civil y de las personas, organizaciones e instituciones que hicieron frente a la represión política, social, cultural y nacional de un régimen ignominioso». Ver http://memoria.gencat.cat/ [consultado el 10 de marzo de 2020].

${ }_{51}$ Ley $4 / 2014$, de 27 de noviembre.

$52 \mathrm{http}: / /$ www.navarra.es/inm/es/ [consultado el 10 de marzo de 2020].

$53 \mathrm{http} / / / \mathrm{www}$. fmomo.org/dedalo/ [consultado el 10 de marzo de 2020]. El propio Instituto Navarro de la Memoria ha optado también por esta plataforma a la hora de gestionar los testimonios de las víctimas, aunque el proyecto no está todavía accesible en abierto. 
tar, de forma multimodal, un determinado tema de interés o quiera conocer toda la información existente sobre una víctima, una localidad o un determinado acontecimiento histórico.

\section{Conclusiones}

A lo largo del presente trabajo nos hemos acercado a distintos contextos y ejemplos en los que se exploraban las posibilidades que nos ofrece la mediación digital a la hora de desarrollar iniciativas de reparación de las víctimas y de recuperación y preservación del patrimonio de la memoria histórica. Lo hemos hecho desde la experiencia de diversas iniciativas que sobre la memoria de las víctimas del franquismo se han llevado a cabo en Navarra, contextualizadas con otros casos más allá de las fronteras de la Comunidad Foral.

Las Humanidades Digitales, cuando se aplican a la gestión pública de la memoria, se acercan también a ese otro espacio de las Public Humanities que tanto desarrollo ha tenido en otros países: un espacio colaborativo de servicio a la ciudadanía, de profundización en los valores de la democracia, de la libertad, de la convivencia sobre la base de la memoria. Las herramientas digitales permiten también, en el caso del mapa de fosas y lugares de memoria, en las investigaciones sobre las personas desaparecidas o en la construcción de un banco digital de testimonios «compartir un conocimiento participativo» y contribuir, al mismo tiempo, a la conformación de una «conciencia pública sobre esos pasados en nuestro presente» ${ }^{54}$. Ese es el alma humanística de proyectos como los analizados en este trabajo, iniciativas que requieren una reflexión ética sobre el papel que desempeña $o$ que debería desempeñar esa suerte de velo que representa lo digital.

En este sentido, hemos visto cómo ese velo digital puede ser la solución a la hora de respetar la voluntad de las familias en conjuntos memorialistas que incorporen el nombre de las víctimas, de modo que pueda haber nombres presentes de forma física, otros que lo estén de manera física y digital, y otros que aparezcan solo en la versión digital. Pero hemos visto también otro tipo de casos en los que el velo digital puede ser contemplado como un intento de ocultamiento de la memoria histórica, relegada a un plano digital al que hay que hacer el esfuerzo de acceder, un acto de voluntad que dificulta en ocasiones el recuerdo colectivo y com-

54 Noiret, 2018, pp. 113-114. 
partido de nuestro pasado. Por ello, al hilo de los casos analizados, podemos afirmar que no hay una única solución aplicable con carácter general, sino que la mediación digital puede requerir de la complementariedad con otras actuaciones en el mundo físico, analógico. Se trata, no lo olvidemos, de cuestiones de primer orden cuando analizamos actuaciones que pretenden un trabajo de memoria desde el respeto al derecho de las víctimas a la verdad, la justicia y la reparación, y al establecimiento de garantías de no repetición, tal y como marcan los organismos internacionales.

Evidentemente, son muchos más y muy variados los ejemplos de mediación digital aplicada al patrimonio de la memoria histórica. Se trata de un ámbito de trabajo en constante crecimiento y transformación. Hay varios proyectos de Humanidades Digitales que están ahora mismo en pleno desarrollo y que son esenciales desde esta preocupación por la forma en que la mediación digital responde a la necesidad de reparación de las víctimas en sociedades como la nuestra. La recreación por medio de realidad aumentada de los campos de concentración y exterminio europeos del siglo $\mathrm{XX}^{55} \mathrm{o}$ de las fosas del franquismo ${ }^{56}$, la aplicación de la imagen en 3D a la preservación de la memoria de los presos del fuerte de San Cristóbal expresada en las inscripciones que hicieron en las paredes de la prisión, etc., son algunos de estos ejemplos.

55 En este sentido caminan algunas de las líneas de trabajo del proyecto Accessing Campscapes: Inclusive Strategies for European Conflicted Pasts (iC-ACCESS): http:// www.campscapes.org/ [consultado el 10 de marzo de 2020]. Como afirma la web del proyecto: «The project team will create a digital network of 4D reconstructed sites through the assimilation of the 3D visualisations and the subsequent layering of documentary evidence (e.g. material traces, oral testimonies, photographs, media, narratives and memories) connected to landscapes, monuments, memorials and museums» (https://www.campscapes. org/research-strands/). Se trata de un aspecto ya explorado por la Future Memory Foundation para el caso del campo de concentración nazi de Bergen-Belsen, en el estado alemán de Baja Sajonia, http://www.futurememoryfoundation.org [consultado el 10 de marzo de 2020]; o por Forensic Architecture para el campo de concentración de Staro Sajmište, en Belgrado, https://forensic-architecture.org/investigation/living-death-camp-staro-sajmiste [consultado el 10 de marzo de 2020]. Otro caso interesante es el del United States Holocaust Memorial Museum y su experiencia con realidad aumentada: https://engage.ushmm. org/2018-augmented-reality.html [consultado el 10 de marzo de 2020].

56 Proyecto en el que está trabajando la Sociedad de Ciencias Aranzadi en el marco de la exhumación de fosas comunes en Navarra por medio del equipo de Francisco Etxeberria y Lourdes Herrasti, concretamente con la colaboración del ingeniero Euken Alonso. Este mismo grupo ha explorado también la aplicación de drones para la fotografía y vídeo digital en el contexto de las exhumaciones. Ver sobre este particular Rodríguez Larrarte, Herrasti y Etxeberria, 2019. 
Por otro lado, en estos últimos tiempos, se está generando también una profunda reflexión sobre el impacto que lo digital está teniendo en ámbitos y momentos tan sensibles para los distintos agentes de la memoria histórica como son las propias exhumaciones ${ }^{57}$. Y son también igualmente relevantes las aportaciones de iniciativas GLAM a la gestión del patrimonio memorialista que han dado lugar a interesantes proyectos desde hace ya varios años.

Mi intención no era, como ya he dicho, elaborar una recopilación exhaustiva de estas aproximaciones. En estas líneas he pretendido llevar a cabo una selección de algunas de estas iniciativas con el ánimo de mostrar cómo las líneas de trabajo de las Humanidades Digitales en el ámbito académico han permeado y calado también en los planos institucional y social en proyectos orientados a la preservación del patrimonio de la memoria, con el objetivo de avanzar en el reconocimiento y la reparación de las víctimas de procesos de vulneración de los Derechos Humanos. Es una gran oportunidad si tenemos presente, además, que la mediación digital en aspectos relacionados con la memoria y su patrimonio está siendo regulada en textos legales de lugares como Navarra (Ley de Lugares de la Memoria Histórica de Navarra) o en documentos de instituciones como la UNESCO (Interpretation of Sites of Memory).

\section{Bibliografía}

Adorno, Theodor W., Dialéctica negativa, Madrid, Taurus, 1992.

AibAR PUENTES, Eduard, «La transformación neoliberal de la ciencia: el caso de las Humanidades Digitales», ArtefaCToS. Revista de Estudios de la Ciencia y la Tecnología, 7.1, 2018, pp. 13-28 (doi: http://dx.doi.org/10.14201/ art2018711328).

Altafaylla, Navarra 1936. De la esperanza al terror, Tafalla, Txalaparta, 2018. BARAIBAR, Álvaro (ed.), «Las Humanidades Digitales desde sus centros y periferias», en Janus. Anexo 2. Humanidades digitales: una aproximación transdisciplinar, 2014, pp. 7-15.

Barrera Becerra, Eduardo, Giráldez Díaz, Javier y Melero Vargas, Miguel Ángel, «Lugares de memoria en Andalucía. Un camino por recorrer», Huarte de San Juan. Geografía e Historia, 27, 2020, pp. 47-70.

57 Especialmente interesante me parece la reflexión que ha hecho Ferrándiz (2018) sobre la forma en que las posibilidades de lo digital han cambiado las prácticas en torno a la gestión de las emociones en el contexto de las exhumaciones de fosas comunes del franquismo. 
BIANCHINI, Maria Chiara, «De la represión al patrimonio: vestigios de la violencia de estado en Madrid y Santiago de Chile», Revista de Dialectología y Tradiciones Populares, 70.2, 2015, pp. 399-426 (doi: https://doi.org/10.3989/ rdtp. 2015.02.005).

Bocanegra Barbecho, Lidia y Toscano, Maurizio, «The Spanish Republican Exile: Identity, Belonging and Memory in the Digital World», en BoROWIECKI, Karol Jan, Forbes, Neil y FreSA, Antonella (eds.), Cultural Heritage in a Changing World, Cham, Springer, 2016, pp. 237-253 (doi: https:// doi.org/10.1007/978-3-319-29544-2).

Comité Económico y Social Europeo, «Dictamen del Comité Económico Europeo sobre "La Senda Paloma de la Paz (White Dove Way) - Propuesta de estrategia de consolidación de la paz de alcance mundial liderada por la UE"», Diario Oficial de la Unión Europea, C228/05, 5 de julio de 2019, pp. 31-36.

De Bernardi, Alberto, «La Casa del Fascio de Predappio», conferencia pronunciada en la I Jornada Internacional «Simbología dictatorial, arte y espacio público», Pamplona, 1 de junio de 2017, http://www.navarra.es/home_es/especial/Agendamemoriahistorica/1junio.htm [consultado 10 de marzo de 2020].

EIroA, Matilde, «La Guerra Civil Española en la actualidad cibermediática», Studia Historica: Historia Contemporánea, 32, 2014, pp. 357-369.

EIROA, Matilde (coord.), Historia y memoria en Red. Un nuevo reto para la historiografía, Madrid, Editorial Síntesis, 2018.

ESCRIBANO GONZÁLVEZ, Elena, «Musealizar la memoria de las víctimas», Revista Historia Autónoma, 12, 2018, pp. 261-278 (doi: https://doi.org/10.15366/ rha2018.12.014).

Fenollosa Tejedor, Pedro y Moncusí Ferre, Albert, «Patrimonialización de la memoria oral e Internet: algunos ejemplos», en XVII Congreso de Estudios Vascos: Gizarte aurrerapen iraunkorrerako berrikuntza = Innovación para el progreso social sostenible (17. 2009. Vitoria-Gasteiz), Donostia, Eusko Ikaskuntza, 2012, pp. 753-768.

FERRÁNDIZ, Francisco, «De la lágrima al píxel. Corrección política y emociones digitales en las exhumaciones de fosas comunes de la Guerra Civil», en Delgado, Luisa Elena, Fernández, Pura y LABAnYi, Jo (coords.), La cultura de las emociones y las emociones en la cultura española contemporánea: (siglos XVIII-XXI), Madrid, Cátedra, 2018, pp. 293-318.

FERRÁndiz, Francisco y BAER, Alejandro, «Digital Memory: The Visual Recording Mass Grave Exhumations in Contemporary Spain», Forum: Qualitative Social Research, 9, 3, 2008, s. p. (doi: http://dx.doi.org/10.17169/fqs9.3.1152).

Ferrándiz Martín, Francisco, Salas Tovar, Ernesto y De Kerangat, Zoe Le Maignan, «De las exhumaciones de fosas comunes a las humanidades digitales», Ponencia presentada en la I Jornada científico-técnica en Humanidades Digitales en el CSIC. Ciencia, tecnología e interdisciplinaridad en la inves- 
Álvaro Baraibar

tigación en Humanidades y Ciencias Sociales, Madrid, 27 de junio de 2017, http://hdl.handle.net/10261/152311 [consultado el 10 de marzo de 2020].

GÁmIR, Agustín, «El giro espacial en las Humanidades Digitales y sus productos cartográficos», Biblio 3W. Revista Bibliográfica de Geografía y Ciencias Sociales, 24, 1275, 2019, pp. 1-27.

García-FunEs, Juan Carlos, «El todo por la parte. La polémica en torno a los nombres de víctimas en el memorial del cementerio de la Almudena», en Hernández Holgado, Fernando y Montero Aparicio, Tomás (eds.), Morir en Madrid (1939-1944). Las ejecuciones masivas del franquismo en la capital, Madrid, Machado Libros, 2020, pp. 135-146.

Gastón Aguas, José Miguel, y Layana Ilundáin, César, «Del terror a la esperanza: lugares de memoria en Navarra», Huarte de San Juan. Geografía e Historia, 27, 2020, pp. 71-94.

Gobierno de NAvarra, Bajo tierra. Exhumaciones en Navarra, 1939-2019, Pamplona, Gobierno de Navarra, 2019.

HERnÀndEZ I MARTí, Gil Manuel, «La memoria oscura: El patrimonio cultural y su sombra», en HeRnÀndEZ I MARTí, Gil Manuel (coord.), VI Congreso Internacional «Restaurar la Memoria»: La gestión del patrimonio: hacia un planteamiento sostenible, 31 de octubre, 1 y 2 de noviembre de 2008, Valladolid, Junta de Castilla y León, 2010, vol. 2, pp. 629-637.

INTERNATIONAL COALITION OF SITES OF CONSCIENCE, Interpretation of Sites of Memory, 2018, https://whc.unesco.org/en/activities/933/ [consultado el 10 de marzo de 2020].

LEY 4/2014, de 27 de noviembre, de creación del Instituto de la Memoria, la Convivencia y los Derechos Humanos, Boletín Oficial del País Vasco, 230, 2 de diciembre de 2014.

LEY 13/2007, del 31 de octubre, del Memorial Democrático, Diario Oficial de la Generalitat de Cataluña, 5006, 12 de noviembre de 2007.

Ley Foral 29/2018, de 26 de diciembre, de Lugares de la Memoria Histórica de Navarra, Boletín Oficial de Navarra, 250, 31 de diciembre de 2018, http:// www.lexnavarra.navarra.es/detalle.asp?r=50972 [consultado el 10 de marzo de 2020].

Mate, Manuel Reyes, La piedra desechada, Madrid, Trotta, 2013.

Mate, Manuel Reyes, «Memoria histórica y ética de las víctimas», Página abierta, 242, 2016, pp. 6-13.

NoIRET, Serge, «Trabajar con el pasado en internet: la historia pública digital y las narraciones de las redes sociales», Ayer, 110.2, 2018, pp. 111-140.

Nora, Pierre, Les lieux de mémoire, Gallimard, Paris, 1984-1992, 3 vols.

Paniagua Santamaría, Pedro, «Memoria en Twitter. La multiplicación del discurso histórico de la violencia», en EIROA, Matilde (coord.), Historia y memoria en Red. Un nuevo reto para la historiografía, Madrid, Editorial Síntesis, 2018, pp. 151-169. 
Mediación digital y memoria histórica: una mirada desde Navarra

PASETTI, Matteo, «Intervista a Alberto De Bernardi sul progetto museográfico per l'ex Casa del fascio e dell'ospitalità di Predappio», e-review. Rivista degli Istituti Storici dell'Emilia Romagna in Rete, 6, 2018, http://e-review.it/pasetti-intervista-a-de-bernardi [consultado el 10 de marzo de 2020].

PRATS, Llorenç, Antropología y patrimonio, Barcelona, Ariel, 1997.

Rodríguez Larrarte, Ángel, Herrasti, Lourdes y ETXEBERria, Francisco, «Uso de drones en arqueología y antropología forense», Revista Internacional de Antropología y Odontología Forense, 1, 2, 2019, pp. 40-45.

SÁNCHEZ CARRETERo, Cristina, «Patrimonialización de espacios represivos. En torno a la gestión de los patrimonios incómodos en España», en ORTIZ, Carmen (ed.), Lugares de represión, paisajes de la memoria. La cárcel de Carabanchel, Madrid, Catarata, 2013, pp. 28-41.

SPENCE, Paul, «Digitally Mediated Memory and the Spanish Civil War», en RIBeIro de Menezes, Alison, Cazorla-SÁnchez, Antonio y Shubert, Adrian (eds), Public Humanities and the Spanish Civil War. Connected and Contested Histories, Cham, Palgrave Macmillan, pp. 189-215.

Traverso, Enzo, El pasado, instrucciones de uso: historia, memoria, política, Madrid, Marcial Pons, 2007.

\section{Datos del autor}

Álvaro Baraibar es doctor en Historia y, actualmente, Profesor del Departamento de Ciencias Humanas y de la Educación de la Universidad Pública de Navarra. Entre octubre de 2015 y agosto de 2019, ha sido Director General de Paz, Convivencia y Derechos Humanos del Gobierno de Navarra y responsable, por tanto, de las políticas públicas de memoria. En sus trabajos sobre Historia cultural de la política ha estudiado aspectos como discurso, identidad, símbolos o la relación entre historia y memoria. Es autor de obras como Extraño federalismo. La vía navarra a la democracia (1973-1982) o Historia y memoria de los símbolos de Navarra. Ha dedicado también su atención a las Humanidades Digitales en obras como Visibilidad y divulgación de la investigación desde las Humanidades Digitales. Experiencias y proyectos o Humanidades Digitales: una aproximación transdisciplinar. En 2014 fue el director del primer congreso sobre Humanidades Digitales en España: Humanidades Digitales: visibilidad y difusión de la investigación. 